\title{
FACTORS THAT AFFECT CLOUD COMPUTING ADOPTION BY SMALL AND MEDIUM ENTERPRISES IN KENYA
}

\author{
John Ngugi Makena \\ Jomo Kenyatta University of Agriculture and Technology \\ Institute of Computer Science and Information Technology \\ P.O. BOX 21403 -00100 Nairobi, Kenya.
}

\begin{abstract}
The number of Small and Medium Enterprises in Kenya have increased tremendously over the last ten years. They have played a profound role in providing employment to the population besides growing the Kenyan economy. Cloud computing is a new entrant to the technology arena which in form of Platform as a Service, Software as a Service and Infrastructure as a Service promises profound reduction in cost of operations in a business. It offers immense benefits as the business enterprise utilizes the pay per use model availed by the cloud service providers as per the needs of the business enterprise. This eliminates the need to purchase expensive software, development platforms and setting up complex ICT infrastructure. This is akin to renting what they need instead of purchasing and owning it.

However, the SMEs in Kenya have not taken up the cloud computing benefits to maximise their competitive advantage. This research paper focuses on the factors that affect the adoption of cloud computing by SMEs in Kenya. The research process involves a descriptive research design. The research findings have shown that technological, organisational and environmental factors have affected the adoption of cloud technologies.
\end{abstract}

Keywords: cloud computing, technological, organisational, environmental.

\section{INTRODUCTION}

Due to severe market competition and dramatically changing business environment, firms have been prompted to adopt various state-of-the -art Information Technologies to improve their business operations. (Pan and Jang, 2008; Sultan, 2010)

In modern technology arena, cloud computing has cut a great and specific niche in businesses. The use of information and communication technologies can improve business competitiveness and has provided genuine advantages for small and medium sized enterprises. (SMEs firms with one to 250 employees (DTI, 2004)), enabling them to compete with large firms (Swash, 1998; Bayo-Moriones and Lera-Lopez, 2007). In Kenya SMEs forms the largest block of employers. They provide the necessary and critical base for economic development. Competition for market share and great profit margins is cut throat. The growth of SMEs is compounded by their agility and adaptability to changing business models.

Cloud computing lowers IT costs and provides organisations with the people and expertise to create an integrated suite of software applications. (Subhankar, 2012)

Some of the promised benefits from cloud computing can be very appealing to SMEs, which need to maximise the return on their investment and still remaining competitive in an ever demanding business environment.(Yazn et al,2012).

The benefits of cloud computing adoption in developing countries have not been thoroughly exploited. If the SMEs adopt the robust services offered by cloud computing, they will have lowered the costs of operation because they would access business application softwares at a low cost. In view of this the research paper main objective is to contribute to a growing body of research on cloud computing by studying the determinants of cloud computing adoption by SMEs in Kenya.
The findings of this research will greatly influence the uptake of cloud services which could effectively and efficiently deliver services which could have otherwise been only accessible by large blue chips corporations and multinationals.

If SMEs have access to scalable technologies they could potentially deliver products and services that in the past only large enterprises could deliver hence flatten the competition arena. (Yazn et al, 2013)

While cloud computing has been discussed as a new technology development that can provide several advantages both strategic and operational to its adopters the cloud computing adoption rate is not growing as fast as expected (Goscinski and Brock,2010).

The SME sector in Kenya has been selected for this study because they are the largest providers of direct and indirect employment hence play a pivotal role in the economic growth of the country.

\subsection{Towards cloud adoption}

A survey on cloud computing awareness by Market Connections (2008) on US defence/ military and Federal government unearthed that cloud utilization is poised for rapid gains as awareness of cloud computing growth. The SME sector in Kenya has adopted various technology solutions that have enhanced their growth. ERPs and other integrated business software systems have been used by big corporates though the SMEs have not managed to access them.

Before any organisation can use cloud computing, it must be aware of existence of such a technology, what it is used for and where it can be applied.(Kiiru,2011).The CEOs of various SMEs in Kenya have adequate awareness of the benefits of technology in their organisations. However, only a few know the benefits of cloud computing beyond the basic description. 
Many have adopted a wait and see attitude as far as adoption of cloud computing is concerned.

According to Ellison (2010), the concept of cloud computing has aroused interest in the enterprise but it is also clear that businesses are testing their options to decide whether they will adopt this technology.

Though there has been increased awareness by cloud services providers on organisational benefits of cloud computing, the rate of adoption is significantly low. A survey conducted by Daniel (2010) titled 'Mobile Technology and Business' in the United States revealed that $95 \%$ of the respondents were generally aware of the cloud computing concept and believe that its role and significance will increase in the next five years. There are various worries from the SMEs about cloud services adoption which contribute to the slow adoption in Kenya.

There have been inadequate research on utilization of technology for business advantage by SMEs in Kenya; various managers expressed concerns of security as a major drawback to the cloud services adoption.

According to Mime Cast (2010), majority of organisations (51\%) in UK and USA using some form of cloud computing service and the levels of satisfaction amongst these organisations is high.

\subsection{How ready are SMEs to adopt cloud computing?}

"Computing services on-demand" is gradually modifying the way information system services are developed, scaled, maintained and paid for. (Yazn et al, 2013). The SMEs readiness to adopt new technology is a determinant of how they will adopt various forms of technology.

They more oftenly have a strong clinch to their former and current existing technologies such that adopting new ones may be difficult decision to make. This is partly due to the perceived losses of existing technology.

From a study carried out in India( Tripathi,2009)with the aim of finding out whether organizations are willing to adopt the technology, the results revealed that cloud computing has not gone past the awareness phase. The research showed that many decision makers are not aware of IaaS, PaaS and SaaS and their differences between public, private and hybrid clouds.

Many SMEs do not want to try a new technology which they are not knowledgeable of due to presumed cost of acquisition and perceived cost of failure. Most of the research and surveys on cloud computing have largely been done in the developed world other than Africa. Hence, the SMEs have scanty references whenever they may need relevant information concerning utilization of cloud services and the rate of success in Africa.

\section{TOE FRAMEWORK}

The TOE is an organisational level theory that provides a framework for technology adoption by an enterprise. It is a theoretical model for cloud computing diffusion needs to consider the weakness in the adoption and diffusion technological innovation which are caused by the specific technological, organisational and environment contexts of the firm. (Chinyao and Mingchang 2011).

Technological context represents the internal and external technologies related to the organisation. The technologies may involve equipment or practice. Organisational context is related to the resources and the characteristics of the enterprise. Environment context refers to the area in which a firm conducts its business which can be related to surrounding elements such as presence of related businesses, competition and availability of other technologies. All the three contexts present both constraints and opportunities for technological innovation and adoption. (Tornatzky and Fleischer, 1990) which influence the technology adoption by firms.

The TOE studies have shown that the following three features influence cloud computing adoption. Technological context(relative advantage, complexity and compatibility);Organisational context(top management support, firm size and technological readiness) and Environmental context(competitive and trading partner pressures).Therefore, TOE provides a clear theoretical basis and a consistent empirical support and the likely outcome of new technology adoption in a firm(Khan and Chau,2001;Zhu et al,2004;Shirish and Teo,2010). Since new technology adoption is a complex issue for many SMEs, this research utilizes the TOE framework as framework that determines the factors that affect cloud computing adoption by SMEs in Kenya.

\section{METHODOLOGY}

\subsection{Research design.}

The research objective is to study the factors that affect cloud computing adoption by SMEs in Kenya. This involves a qualitative study of the factors and their extent of influence based on the TOE framework.

\subsection{Sampling and data collection}

A total of 220 interviews with ICT officers and business owners distributed proportionately were carried out. However, a total of 260 contacts were made which resulted to 202 interviews. This resulted to a response rate of $78 \%$.Among the 202 respondents, $55 \%$ were ICT officers who were mandated with making key decisions in the firm while $45 \%$ were owners of the business enterprises. Questionnaires were used to collect primary data through interviews. The respondents were owners and ICT officers. The data obtained was analysed using descriptive statistics.

\section{RESULTS AND DISCUSSION}

This research was greatly guided by the TOE framework. The TOE framework has been credited and proposed by Shirish and Teo (2010); Lin and Lin (2008) as precise approach towards analysing IT adoption by firms.

From the findings of the research, it emerged that up to $70 \%$ of the respondents were aware of the presence of cloud computing technology in the modern business environment. About 50\% were aware of the benefits of the technology to their specific business model. However, $30 \%$ of the respondents never expected any benefits of adopting the technology.25\% of the respondents were willing to switch 
their current technology to cloud platforms while $60 \%$ were not willing to abandon their current technology and adopt cloud computing model.50\% expressed their desire to adopt cloud computing technology gradually in the next five years.

\subsection{Environmental context}

Trading partner and competitive pressures played a significant role in determining whether to adopt cloud computing. From the research findings, it was observed that environmental context was influenced by the nature of the business. For example effects of environmental context on firms involved in manufacturing were different from those involved in services industry. This is because firms on same business activity were competing with each other. If one has not embraced technology, then others did not find solid reason to adopt cloud computing. However, those in sectors that technology was key were willing to adopt cloud computing so as to be more competitive. Their customers also put pressure on these firms causing a positive effect on the technology adoption. However, these pressures were not leading to a compulsory acquisition of technology since the customers received the services they wanted using current set up. These findings show that firms are aware of technological needs to enhance proper delivery of services but they choose not to shift to that technology. However, when they are pressurised by competition they aggressively adopt such technologies swiftly. This revelation is consistent with earlier studies from Chong and Ooi (2008) and Oliveira and Martins (2010) and implies that when firms are faced with intense competition, they tend to implement changes more aggressively. For example Wal-Mart, which requires its partners to either adopt radio frequency identification or lose their business (Chong and Ooi, 2008).

From the findings, it is clear that firms need to be made aware of specific benefits of adopting cloud services in the areas of business operations. This will result to a profound plan for adoption since the businesses will be aware of cost implications and expected profits.

\subsection{Organisational context}

The top management support was found to be a significant factor in the cloud adoption. This research found out that top managers were not willing to invest resources to new technology when there is an existing and working technology. However, some managers unwillingness to adopt was as a result of scanty knowledge they have about cloud computing. They cited that it is a new technology hence a'wait and see" approach was the best for them. These findings are consistent with those of Lertwongsaien and Wongpinunwatana (2003) and Ramdani and Kawaleki (2007, 2008) who found that without top management support, SMEs are less likely to adopt new technologies.

The technology readiness of the firm also affected the adoption of cloud computing technologies. Firms with fewer employees with adequate ICT knowledge were generally not ready with adoption as opposed to those whose employees were having adequate ICT skills. For example technology based firms were willing to adopt the technology.

Small firms also expressed their desire to adopt the technology while larger ones were less willing to change. This could be attributed to the fact that decision making is easier in a small than the long and tedious protocol based decision making process in large organisations. This is consistent with Chinyao and Mingchang (2011) that organisational competency and readiness may help to leverage existing information technology applications and data resources across key processes along the value chain when the firms embeds the cloud computing service.

\subsection{Technological context}

Benefits that a firm expects to accrue from a certain technology motivates it to adopt it because employee appreciation of the relative advantages of the adopted system to raise work efficiency. Cloud computing has an advantage of increased efficiency in provision of services. The relative advantage of cloud computing services implementation could improve speed of business communications, efficiency of coordination among firms, customer communications and access to market information mobilisation.(Ambrust et al,2010).The findings of this research showed that the respondents were aware of the relative advantages of cloud computing. However, their inadequate knowledge about the cloud concept proved to be a major drawback towards making decisions pertaining adoption. Though the relative advantage was clear to most of the respondents, they cited cost of implementation is high. Therefore, this is consistent with Teo et al (2009) who cited that cost of implementing new technology systems, the cost of the systems themselves can be comparatively high and often represent a major barrier to their adoption.

The complexity of the proposed cloud services and compatibility were also points of concern by many respondents. However, these were not primary factors to consider when adopting such technology. The findings showed that several respondents believed that there will be seamless compatibility with their current technology or with few manageable modifications of their current systems. These findings were inconsistent with Oliveira and Martins, (2010).This findings could be due to the fact that many respondents were ICT officers who were generally conversant with operations of networks and databases. They may have been involved in previous compatibility issues and complexity constraints involving other technologies. However, this does not translate to adequate understanding of cloud technology like how to set up a private cloud. Therefore, tough complexity and compatibility did not appear as a general point of concern, it could still pose a challenge in adoption of cloud computing.

\section{CONCLUSION}

The dominant paradigm in studying IT adoption involves identifying contingency factors that facilitate or hinder the adoption decisions in an organisation.(Fichman,2004;Troshaniet al,2011).Though adoption of new technology is a complicated affair, the different factors in technological, organisational and environmental context vary across different innovations.(Bakers,2011).

The cloud service providers have an enormous task of creating awareness among all players in the business environment so that they can critically assess their internal structures and review them in readiness to adopt the new technology. Cloud computing is a relatively new phenomenon which have great potential of decreasing business operation costs and increase their efficiencies. The TOE framework has 
provided an elaborate assessment criterion of factors influencing cloud adoption and it has shown that firms need to firm up their organisational, technological and environmental contexts so as to readily incorporate the new technology.

According to Yazn et al(2011) the nature of cloud computing should offer enough scope to generalize findings beyond the geographical area of study and to SMEs in other regions and even countries as cloud computing transcends boundaries and regional ICT infrastructure is not considered a major obstacle for the adoption process. The SME sector in Kenya has to position itself and align their objectives towards adoption of cloud computing as a new technology entrant in the market. The adoption of computing services on demand through a pay per use model will provide the SMEs sector with a wide pool of resources that befits their business model at a lower cost. Since the goal of any SMEs is to maximise profits, the resources saved after adopting cloud computing will be useful in expansion, research, improving the welfare of their employees and provision of quality services to customers.

In Kenya, technology advancements have fairly penetrated various markets. Therefore it will not be an uphill task to get personnel with technological prowess and appropriate technology base of running cloud applications over large networks. In future, further research could build on this study and find out qualitatively and quntitavely factors that affect cloud adoption by specific industries. Various stakeholders like cloud service providers, policy makers in Government should also be include in future research. SMEs sector in other countries could also be studied since all over the world the SME sector play profound role in economic growth. Therefore, their adoption of cloud computing and other relevant futuristic technologies will continuously position them in fast lane of growth and prosperity.

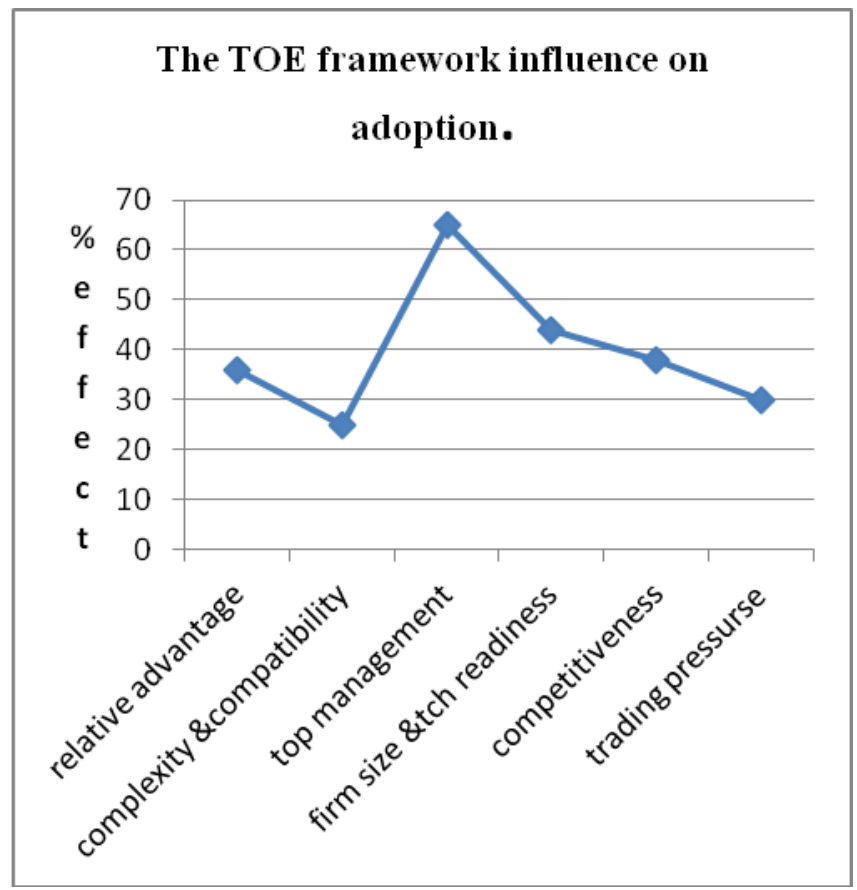

\section{ACKNOWLEDGEMENTS}

I humbly thank all professionals in the technology and SME sectors for their profound input in this research. I also thank
[8] Krutz, R.L. and Vines, R.D. (2010), Cloud Security: A Comprehensive Guide to Secure Cloud Computing, Wiley, New York, NY.

all my colleagues and family for their tireless support in the process of this research.

\section{REFERENCES}

[1] Buyya R., C. S. Yeo, S. Venugopal, J. Broberg, and I. Brandic, "Cloud computing and emerging IT platforms: Vision, hype, and reality for delivering computing as the 5th utility," Future Gener.Comput Syst., vol. 25, pp. 599-616, 2009.

[2] Cena F., Farzan R., Lops P., Web 3.0: Merging Semantic Web with Social Web,

Proceedings of the20th ACM conference on Hypertext and hypermedia, HT'09, June 29-Jul1, 2009 , page 385 .

[3] Dorey P. G. and A. Leite, "Commentary: Cloud computing - A security problem or solution? "Information Security Technical Report, vol. 16, pp. 89-96, 2011

[4] Erdogmus H., "Cloud Computing: Does Nirvana Hide behind the Nebula?" IEEE Software., vol.26 pp. 4-6, 2009.

[5] Kiiru, W. K. (1991). A review of the institutional lending to the jua kali and small enterprise sector in Kenya. Geneva: International Labour Organisation.

[6] King, K. \& McGrath S. (2002) Globalisation, Enterprise and Knowledge:

Educational Training and Development, International Review of Education, Vol. 50(1).

Khajeh-Hosseini A., D. Greenwood, and I. Sommerville, "Cloud Migration: A Case Study of

Migrating an Enterprise IT System to IaaS," presented at the Proceedings of the 2010 IEEE 3rdInternational Conference on Cloud Computing, 3rdinte

Lillard T. V., C. P. Garrison, C. A. Schiller, and J. Steele, "Chapter 12 - The Future of Cloud Computing," in Digital Forensics for Network, Internet, and Cloud Computing, Boston: Syngress, 2010, pp. 319-339.

Longenecker, J. G., Petty, C. W., Moore, J. W. and Palich, L. E. (2006). Small Business Management, An entrepreneurial emphasis. London: Thomson

Marston S., Z. Li, S. Bandyopadhyay, J. Zhang, and A. Ghalsasi, "Cloud computing - The Business perspective," Decision Support Systems, vol. 51, pp. 176-189, 2011.

Moyer, C.M. (2011), Building Applications in the Cloud: Concepts, Patterns, and Projects, AddisonWesley Professional, Reading, MA.

Saul Berman L. K.-T., Anthony Marshall and Rohini Srivathsa, "The power of cloud-Driving Business model innovation," IBM Institute for Business Value, Feb 2012 South Western. 
International Journal of Computer Applications Technology and Research

Volume 2- Issue 5, 517 - 521, 2013, ISSN: 2319-8656

[14] Shayan J., A. Azarnik, S. Chuprat, and M. Zamani, computing in Educational environment," 2012.

"Identifying security risks of exploiting Cloud 